\title{
Intravenous Repetitive Injection of Lidocaine Associated with Pregabalin Enhances Oxidative Stress Parameter in Fibromyalgia Patients' Blood: A Randomized Trial
}

\author{
José Barreto Cruz Nogueira, Luana Heimfarth, Vera Maria Silveira de Azevedo and Lucindo \\ José Quintans Júnior* \\ Laboratory of Neurosciences and Pharmacological Tests (LANEF), São Cristóvão-SE, Brazil
}

*Corresponding author: LJ Quintans-Júnior, Department of Physiology (DFS), Laboratory of Neuroscience and Pharmacological Assays (LANEF). Federal University of Sergipe (UFS), Av. Marechal Rondom, s/n, São Cristóvão, Sergipe-Brazil

\section{ARTICLE INFO}

Received: 幽 September 21, 2021

Published: 幽 October 01, 2021

Citation: José Barreto Cruz Nogueira, Luana Heimfarth, Vera Maria Silveira de Azevedo and Lucindo José Quintans Júnior. Intravenous Repetitive Injection of Lidocaine Associated with Pregabalin Enhances Oxidative Stress Parameter in Fibromyalgia Patients' Blood: A Randomized Trial. Biomed J Sci \& Tech Res 39(2)-2021. BJSTR. MS.ID.006261.

Keywords: Lidocaine; Glutathione; Oxidative stress; Fibromyalgia; Pain

\begin{abstract}
Background: Fibromyalgia syndrome (FMS) is an extra-articular rheumatologic disease with an estimated prevalence of $2-4 \%$, whose etiology and pathogenesis are still unknown. Oxidative stress is an imbalance between products of oxidation and antioxidant defenses and plays a critical role in the etiopathogenesis of FMS. Lidocaine is a local anesthetic with rapid onset of action, safety profile, low cost and wide availability that can reduce nerve signal conduction and consequently attenuate pain. Intravenous repetitive injections have been used to manage chronic pain.
\end{abstract}

Purpose: This study aimed to investigate the effects of repetitive intravenous lidocaine injections associated with pregabalin on oxidative and antioxidant parameters in women with FMS.

Methods: Oxidative stress was determined by measuring the levels of GSH, lipoperoxidation, catalase, and superoxide dismutase in the blood of 48 female patients with FMS who were treated with lidocaine or saline. The FIQ and VAS valued clinical parameters of FMS as instruments of valuation of pain.

Results: Regarding the Visual Analog Scale (VAS) and Fibromyalgia Impact Questionnaire (FIQ) scores, patients treated with lidocaine/pregabalin (GPL) revealed improvements in the parameter analyzed compared to the initial and final treatment time. Moreover, lidocaine administration significantly increased GSH levels $(p<0.001)$ and non-enzymatic capacity (FRAP, $\mathrm{p}<0.01$ ) in blood and plasma and decreased lipoperoxidation in blood cells without altering sulfhydryl groups comparing the first and second times of treatment.

Conclusion: Lidocaine plus pregabalin significantly stimulated antioxidant defenses and reduced oxidative stress.

\section{Introduction}

Fibromyalgia syndrome (FMS) is a clinically well-defined disease of extra-articular rheumatologic origin with an estimated $2-4 \%$ prevalence. It is the most found chronic musculoskeletal pain condition, and its etiology is still unknown [1]. Its symptoms also include non-recoverable sleep patterns, cognitive dysfunction, headache, morning stiffness, fatigue, depression, and anxiety [2]. Oxidative stress is an imbalance between oxidation products and antioxidant defenses and has recently been associated with several 
events in the pathogenesis of FMS $[3,4]$. Patients with FMS have decreasedlevels of glutathione (GSH) and increased lipoperoxidation in blood and plasma, which seems to be associated with a worsening of patients' clinical condition [5]. The pathology of fibromyalgia (FM) causes a negative regulation of catalase activity in patients' erythrocytes and leukocytes [6,7]. Research has also described the effects of antioxidant redox systems, levels of protein carbonylation and lipoperoxidation on the pathogenesis of FMS, and increased oxidative stress is strongly associated with the FMS severity [5]. Thus, managing the oxidative profile can be a promising approach to optimize the FMS treatment, although there is a little-explored gap in this profile. There is still no specific pharmacological therapy to date to relieve such syndrome, and the currently available drugs are used to manage symptoms. Although there are drugs that can treat FMS symptoms, such as neuromodulators, antidepressants, and muscle relaxants, some side effects and their low efficacy have been reported to limit therapeutic adherence. Lidocaine is an aminamide-type local anesthetic with fast onset, safety profile, low cost, and wide availability. It inhibits neuron-dependent voltagegated sodium channels, reducing the nervous signal conduction and consequently blocking pain. Intravenous lidocaine showed positive results in treating acute and chronic neuropathic pain syndromes, such as trigeminal neuralgia and peripheral nerve damage. However, the role of intravenous lidocaine injection in FMS needs to be clarified.

Pregabalin was the first drug approved by the Food and Drug Administration (FDA) to treat FMS. Its structure is similar to the gamma-aminobutyric acid (GABA) neurotransmitter, without pharmacological action in this way, but in voltage-dependent calcium channels [8]. Few studies have reported using lidocaine with pregabalin to manage FM, and we have not found reports of studies valuating the oxidation parameters of FM patients with this treatment. Therefore, the hypothesis that there could be an effect on oxidative stress allowed us to assess its correlation with oxidative symptoms and parameters.

\section{Methodology}

\section{Ethical Aspects}

The Ethics Committee of the Federal University of Sergipe (UFS) approved the clinical study opinion No. 2.637.928 (Certificate of Presentation for Ethical Consideration (CAAE) protocol No. 85503418.2.0000.5546). All subjects who volunteered for the trial were women included in the study only after signing the free and informed consent form. The Universal Trial Number (UTN) is U1111-1257-3477. ReBEC trial: (req:10410) Benefits of innovative treatment for women with fibromyalgia: a procedure to follow.

\section{Experimental Groups and Schematic Design of Experiments}

Forty-eight female patients who met the 2016 American College of Rheumatology (ACR) FMS classification criteria were enrolled. The inclusion/exclusion criteria adopted in the study excluded patients with other comorbidities such as epilepsy, recent trauma ( $\leq 3$ months), psychiatric and rheumatic disorders, moderate or severe neuromuscular disorders, hypothyroidism or hyperthyroidism, infectious arthroplasty, other chronic pain syndromes, hypersensitivity to drugs, and neoplasms disorders.

Twenty-four patients formed two groups randomly.

Pregabalin Group (GP): Women taking $150 \mathrm{mg}$ pregabalin daily and submitted to a hospital procedure performed at the surgical center for an intravenous administration of $0.9 \%$ physiological solution for three consecutive weeks.

Pregabalin/lidocaine Group (GPL): Women taking $150 \mathrm{mg}$ pregabalin daily and submitted to a hospital procedure performed at the surgical center for intravenous administration of lidocaine (3 mg.kg) for three consecutive weeks.

FM patients valuated for five weeks. The experimental procedure schematic design shown in Figure 1. The lidocaine infusion was applied at the third (T2), fourth (T3), and fifth (T4) weeks. The FIQ and VAS questionnaires were performed at the first (T0), second (T1), third (T2), fourth (T3), and fifth (T4) weeks.

All patients took pregabalin (150 mg.kg) for treatment as the therapeutic protocol of the HU-UFS outpatient clinic. Thus, since our research ethics committee did not authorize an experimental group of fibromyalgia patients treated with lidocaine alone, they used the previous pregabalin because it is a pain clinic, which is a limitation of this study Table 1 and Table 2.

\section{Fibromyalgia Impact Questionnaire (FIQ score)}

The FMS functional status was valuated by the FM impact questionnaire (FIQ). The questions aimed to highlight well-being and the loss of daily work, difficulty to work, fatigue, pain, and well-being in the morning after waking up, stiffness, anxiety, and depression. High scores showed functional limitations [9].

\section{Intensity of Resting Pain (VAS score)}

A numerical scale of 11 points measured the intensity of the pain according to how intense patients report the pain to be, in which 0 is considered painless and 10 is the worst pain. The test is an analogic visual scale completed by the patients themselves [10]. The FMS functional status was valuated by the FM impact questionnaire (FIQ). The questions aimed to highlight well-being 
and the loss of daily work, difficulty in working, fatigue, pain, and well-being in the morning after waking up, stiffness, anxiety, and depression. High scores showed functional limitations [9]. The visual analog scale (VAS) is a subjective measure validated for acute and chronic pain. Patients reported their scores by making a handwritten mark on a $10 \mathrm{~cm}$ line representing a continuum between "no pain" and "worst pain".

\section{Biochemical Measures}

\section{Blood Collection and Preparation of Blood Samples}

During the second and third trials, corresponding to the second and third weeks, venous blood samples of all individuals were collected in test tubes with no anticoagulant or EDTA. Fresh whole blood samples were separated and used for determined reduced glutathione (GSH) and lipoperoxidation. A portion of EDTA-blood was centrifuged for 10 minutes at $3000 \mathrm{xg}$ to separate plasma and erythrocytes that were then washed three times with PBS and kept frozen at $-80^{\circ} \mathrm{C}$ until analysis for activities of RBC antioxidant enzymes, reduced sulfhydryl groups, and ferric reducing ability of plasma (FRAP).

\section{Ferric-Reducing Ability (FRAP assay)}

FRAP level in plasma was measured according to the method by Benzie and Strain, as described previously [11]. The plasma samples were mixed with a reagent mixture containing acetate buffer (pH 3.6), $5 \mathrm{mM}$ tripyridyltriazine in $40 \mathrm{mM} \mathrm{HCl}$, and $20 \mathrm{mM}$ ferric chloride. Absorbance was assessed at a $593 \mathrm{~nm}$ wavelength. FRAP values were expressed as $\mu \mathrm{g} / \mathrm{mg}$ protein.

\section{Reduced Sulfhydryl Groups and GSH Determination}

To measure the levels of reduced thiol (-SH) groups in protein and nonprotein fractions from plasma, an $80 \mu \mathrm{g}$ sample aliquot reacted with a $10 \mathrm{mM}$ 5,5- dithionitrobis 2-nitrobenzoic acid. After 60 minutes of incubation at room temperature, the absorbance was read in a spectrophotometer set at $412 \mathrm{~nm}$ [12].

\section{Lipid Peroxidation Level Determinations}

Lipoperoxidation levels in the total blood samples were measured with the thiobarbituric acid reaction according to the method by (Draper \& Hadley 1990 [13].

\section{Catalase Activity}

Catalase activity was assayed in blood cell lysates by measuring the hydrogen peroxide $\left(\mathrm{H}_{2} \mathrm{O}_{2}\right)$ absorbance decrease ratio in a spectrophotometer at $240 \mathrm{~nm}$ [14].

\section{Superoxide Dismutase Activity}

Superoxide dismutase (SOD) activity was determined in blood cell lysates from the inhibition of superoxide anion-dependent adrenaline autoxidation in a spectrophotometer at $480 \mathrm{~nm}$ as previously described [15].

\section{Statistical Analysis}

The results were expressed as a mean \pm S.E.M or mean \pm S.D. Samples were assessed for normal distribution according to the Shapiro-Wilk test. Differences between the two groups were analyzed using the Mann-Whitney $U$ test for independent samples or the Wilcoxon test for dependent samples. The rank-biserial correlation was used as a side effect. Differences were considered significant if $\mathrm{p}<0.05$. The statistical analyses were made using the GraphPad Prism ${ }^{\circledR} 5.0$ software (GraphPad Prism Software Inc., San Diego, CA, USA).

\section{Sample Size Calculation}

Although a 1:1 proportion for randomization was proposed, the final proportion was 3:2 due to a lack of follow-up. Thus, for a significant difference at the $5 \%$ level with $80 \%$ power, with a very large effect size (Cohen's D = 1.2), 25 patients with a 3:2 ratio are necessary, considering a correction of asymptotic relative efficiency for non-parametric tests.

\section{Results}

According to the study flowchart, the steps occurred as shown in Figure 1.

Study flowchart

The description of the patients is reported in Figure 2.

No difference was found in demographic values for patients with FM treated with pregabalin (GP) or treated with pregabalin/ lidocaine (GPL) (Table 1).

Table 3 Regarding the VAS scores, patients treated with lidocaine/pregabalin (GPL) showed improvements in the parameter analyzed compared initial (T0) and final time (T5) of treatment. No difference was found in patients from the group pregabalin (GP). Besides, a considerable improvement also was seen in the FIQ score compared initial (T0) and final (T5) time of vasluation in GPL and GP groups. No difference was reported between the GPL and GP groups in VAS, neither the FIQ score in the final time (T5) (Table 2).

Table 4 The effect of repetitive intravenous lidocaine 3 mg.kg.h, diluted in $250 \mathrm{ml}$ of $0.9 \%$ saline through an infusion pump, administration associated with pregabalin (150 mg.kg) on the redox status are described in Figures 2 and 3. The blood samples were collected before first lidocaine administration (at the third week (T2)) and after the third lidocaine infusion (at the fifth week (T4)), and the analyses was performed at $\mathrm{T} 2$ and $\mathrm{T} 4$ in the same patient from the GPL (lidocaine/pregabalin) and GP groups (pregabalin) (Figure 1). 
Table 1: Demographic features of patients.

\begin{tabular}{|c|c|c|c|c|}
\hline & GPL $(\mathbf{n}=18)$ Mean \pm SD & GP $(\mathbf{n}=\mathbf{1 7})$ Mean \pm SD & p-value & R \\
\hline Age $($ years $)$ & $49.17 \pm 6.16$ & $45.82 \pm 7.67$ & 0.141 & 0.294 \\
\hline BMI $\left(\mathrm{kg} / \mathrm{m}^{2}\right)$ & $26.74 \pm 4.79$ & $27.7 \pm 4.41$ & 0.488 & -0.141 \\
\hline
\end{tabular}

Note: BMI, body mass index; GPL, pregabalin/lidocaine group; GP, pregabalin group; R, Rank-Biserial Correlation. Values are expressed in means \pm SD. Mann-Whitney Test.

Table 2: Intensity of resting pain (EVA score) and Impact of Fibromyalgia (FIQ score) determined in initial time (T0) and final time (T5).

\begin{tabular}{|c|c|c|}
\hline \multicolumn{1}{|c|}{ GPL (n=18) Mean \pm SD } & GP (n=17) Mean \pm SD \\
\hline \multicolumn{1}{|c|}{ EVA score } & $7.71 \pm 1.11$ \\
\hline T0 & $8.33 \pm 1.0$ & $6.13 \pm 2.17$ \\
\hline T5 & $4.89 \pm 1.05$ & 0.141 \\
\hline p-value & 0.0005 & 0.464 \\
\hline R & 0.975 & $80.79 \pm 12.7$ \\
\hline T5 & FIQ score & $57.55 \pm 20.09$ \\
\hline $\mathrm{p}$-value & $80.11 \pm 9.1$ & 0.024 \\
\hline R & $57.55 \pm 21.12$ & 0.629 \\
\hline
\end{tabular}

Note: BMI, body mass index; GPL, pregabalin/lidocaine group; GP, pregabalin group; R, Rank-Biserial Correlation; T0, initial time; T5, final time. Values are expressed in means \pm SD. Mann-Whitney Test

Table 3: Demographic features of patients.

\begin{tabular}{|c|c|c|c|c|}
\hline & GPL $(\mathbf{n}=\mathbf{1 8})$ Mean \pm SD & GP $(\mathbf{n = 1 7})$ Mean \pm SD & p-value & R \\
\hline Age $($ years $)$ & $49.17 \pm 6.16$ & $45.82 \pm 7.67$ & 0.141 & 0.294 \\
\hline BMI $\left(\mathrm{kg} / \mathrm{m}^{2}\right)$ & $26.74 \pm 4.79$ & $27.7 \pm 4.41$ & 0.488 & -0.141 \\
\hline
\end{tabular}

Note: BMI: Body mass index; GPL: Pregabalin/lidocaine group; GP: Pregabalin group; R: Rank-biserial correlation. Values are expressed in means \pm SD. Mann-Whitney U Test.

Table 4.

\begin{tabular}{|c|c|c|}
\hline \multicolumn{1}{|c|}{ GPL (n=18) Mean \pm SD } & GP (n=17) Mean \pm SD \\
\hline \multicolumn{1}{|c|}{ EVA score } & $7.71 \pm 1.11$ \\
\hline T0 & $8.33 \pm 1.0$ & $6.13 \pm 2.17$ \\
\hline T5 & $4.89 \pm 1.05$ & 0.141 \\
\hline p-value & 0.0005 & 0.464 \\
\hline R & 0.975 & $80.79 \pm 12.7$ \\
\hline T0 & FIQ score & $57.55 \pm 20.09$ \\
\hline T5 & $80.11 \pm 9.1$ & 0.024 \\
\hline p-value & $57.55 \pm 21.12$ & 0.629 \\
\hline R & 0.0078 & \\
\hline
\end{tabular}

Note: BMI: Body mass index; GPL: Pregabalin/lidocaine group; GP: Pregabalin group; R: Rank-biserial correlation; T0: Initial time; T5: Final time. Values are expressed in means \pm SD. Mann-Whitney U Test. 


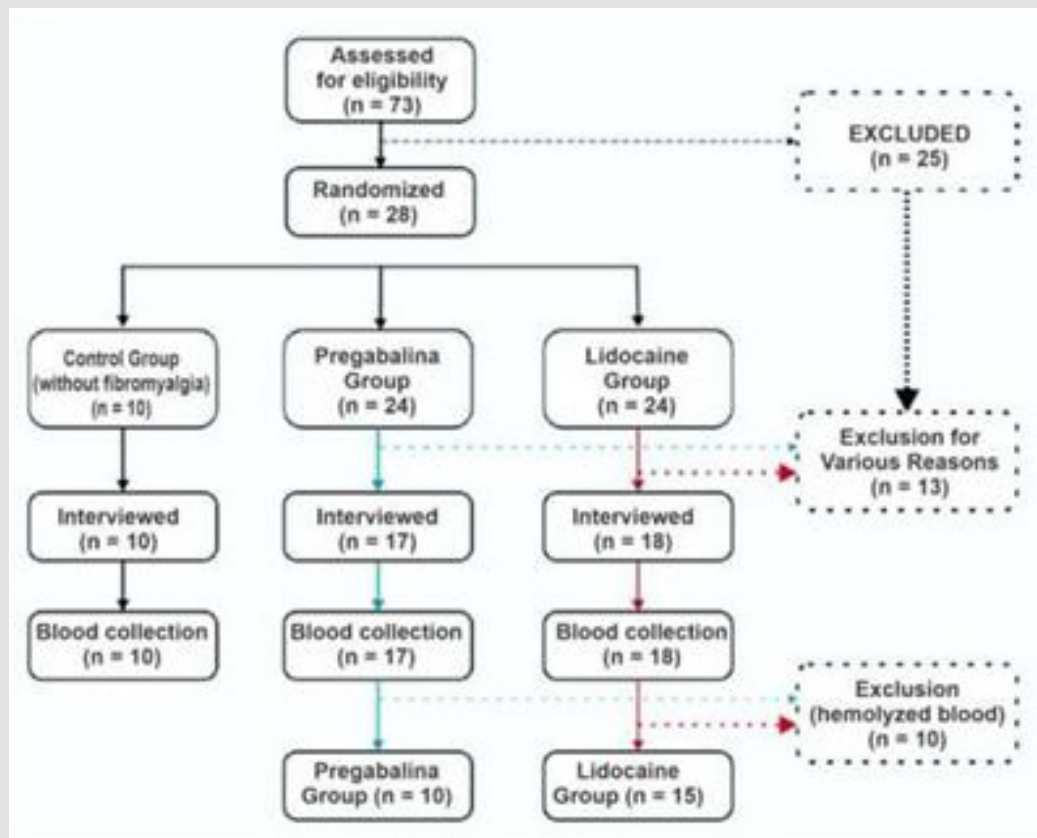

Figure 1.

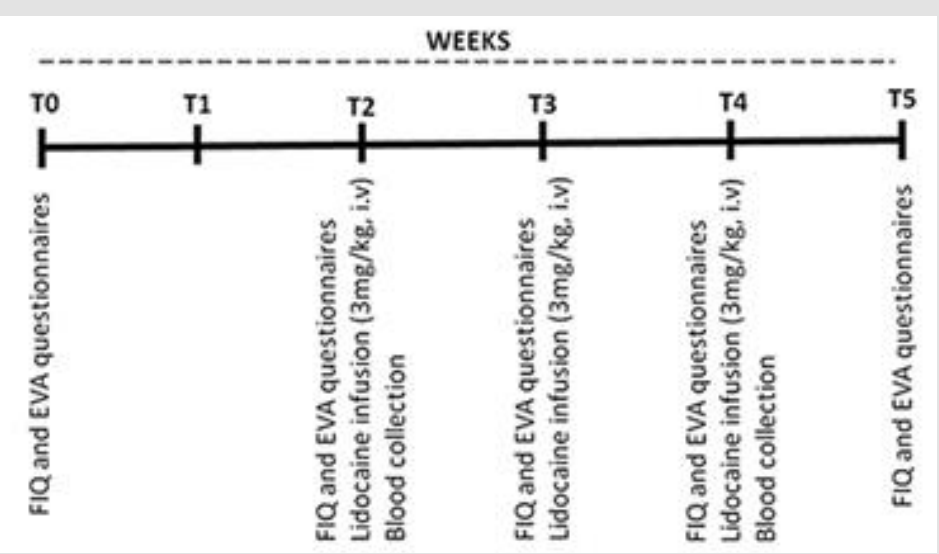

Figure 2: Schematic representation of experimental procedures.

Figure 2 represents the oxidative damage and non-enzymatic antioxidant profile. The results showed that lidocaine/pregabalin administration attenuates the redox damage caused by FM. We found that lidocaine/pregabalin increased GSH levels $(\mathrm{p}<0.01)$ and non-enzymatic capacity (FRAP) $(\mathrm{p}<0.05)$ in blood and plasma of the GPL group (Figure 2A \& 2B). Moreover, lidocaine decreases lipoperoxidation $(\mathrm{p}<0.001)$ in blood cells of the GPL group (Figure 2C) without changing the sulfhydryl groups (Figure 2 D). No changes were found in the GP group (pregabalin) between T2 and
T3 in oxidative damage and non-enzymatic antioxidant status.

Figure 3 describes the effect of lidocaine/pregabalin administration on enzymatic antioxidant defense catalase and superoxide dismutase. Lidocaine/pregabalin could not alter enzymatic antioxidant activities in blood cells in both the GPL and GP groups (Figure 3).

Figure 4 Mild headache, dizziness, and drowsiness have been reported. 
A

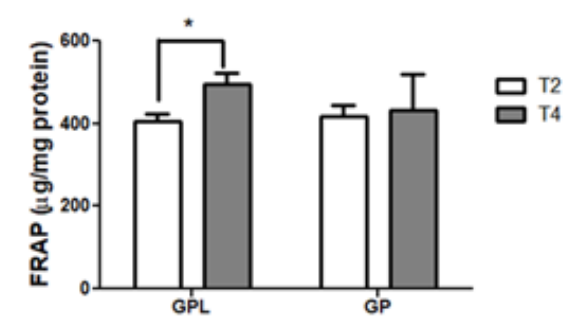

c

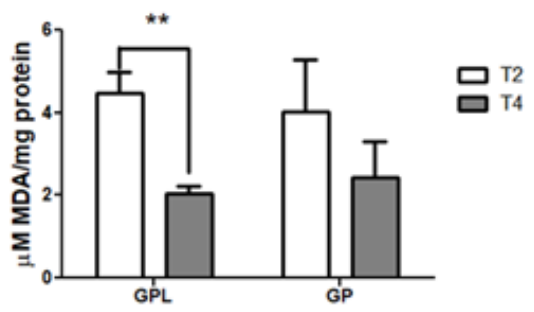

B

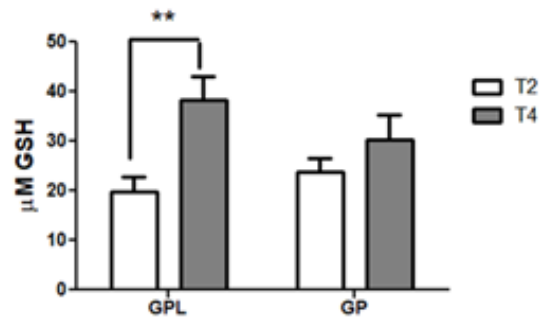

D

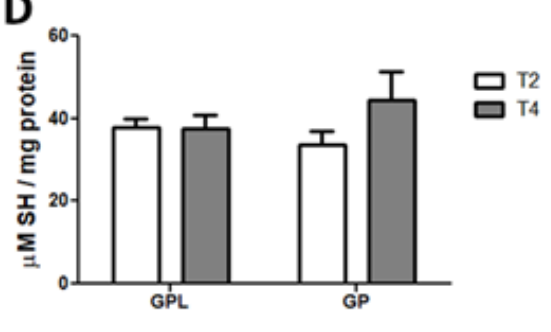

BMI: Body mass index; GPL: Pregabalin/lidocaine group; GP: Pregabalin group; R: Rank-biserial correlation. Values are expressed in means $\pm \mathrm{SD}$. Mann-Whitney U Test.

Figure 3: Effect of repetitive intravenous injection of lidocaine ( $3 \mathrm{mg} . \mathrm{kg}$ IV) on parameters of oxidative imbalance in blood cells and plasma of FM patients. Ferric-reducing ability (FRAP) (A); GSH levels (B); Thiobarbituric acid reactive substance (TBARS) (C); Total reduced thiol content (SH) (D). Data are reported as mean \pm SEM of 15 patients (GPL group) and ten patients (GP group). Statistically significant differences from T2 (before lidocaine injection) and T4 (after third lidocaine injection) as determined by the Wilcoxon test. GPL: Pregabalin/lidocaine group; GP: Pregabalin group.

A

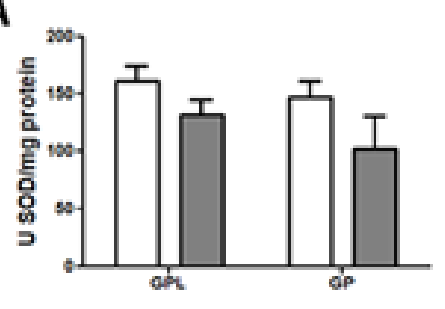

B

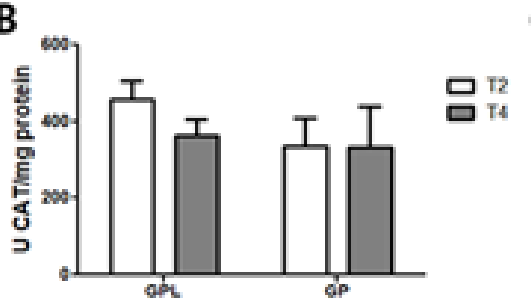

C

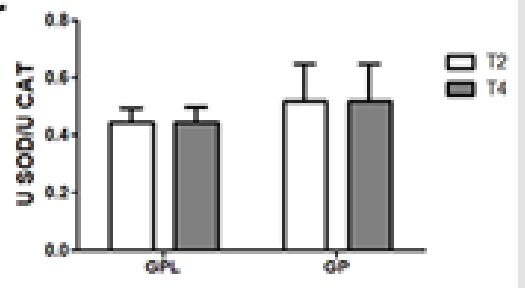

Figure 4: Effect of repetitive intravenous injection of lidocaine ( $3 \mathrm{mg} / \mathrm{kg}$ IV) on enzymatic antioxidant defenses in FM patients' blood cells. Superoxide dismutase activity (A); Catalase activity (B); SOD and CAT ratio (C). Data are reported as mean \pm SEM of 15 patients (GPL group) and ten patients (GP group).

Statistically from T2 (before lidocaine injection) and T4 (after third lidocaine injection) as determined by the Wilcoxon test. GPL: Pregabalin/lidocaine group; GP: Pregabalin group.

\section{Discussion}

This study investigated the beneficial effects of repetitive intravenous injection of lidocaine associated with pregabalin on FM and the role of oxidative stress in the lidocaine/pregabalin pharmacological action. There were significantly improved VAS and FIQ scores in the GPL group (lidocaine/pregabalin). Moreover, to the best of our knowledge, our findings also showed for the first time that lidocaine/pregabalin attenuated the oxidative stress caused by FM, especially by regulating the non-enzymatic antioxidants defense. Lidocaine is a classical local anesthetic and antiarrhythmic drug that changes neuron depolarization by blocking the fast voltage-gated sodium $\left(\mathrm{Na}^{+}\right)$channels. It is also used as an analgesic for several painful conditions [16]. Nowadays, several works have described the effect of lidocaine in attenuating fibromyalgia symptoms. Intravenous lidocaine administration inhibits the pain caused by a deep ischemic but not the superficial cutaneous pain modalities or tactile sensation [17]. Also, repeated lidocaine injections into myofascial points attenuated clinical FM pain and several tender points $[8,17]$, and a single administration of lidocaine into the trapezius muscle reduced secondary hyperalgesia in FM patients [18]. Our results showed that intravenous injections 
of lidocaine associated with pregabalin (orally) reduced the pain, which is supported by the VAS and FIQ scores. Chronic pain is one of FMS's main symptoms and modulating that parameter could lead to a better quality of life. Moreover, our findings also showed improved functional and physical capacity and mental health status assessed by the FIQ score in the pregabalin/lidocaine group.

Thus, administering lidocaine/pregabalin modulates morning tiredness, stiffness, anxiety, pain, and depression in FM patients, thus decreasing FMS symptoms [19]. However, no differences were found between the GPL and GP groups regarding the FIQ scores. Such finding agrees with a study by Oliveira [20], which showed that clinical pain was attenuated after lidocaine treatment. The effect was similar to that of saline administration, which indicated that the additional factor to overall analgesia could be inferred in the lidocaine effect. Additionally, the pain parameters improved in the group treated with lidocaine/pregabalin, reinforcing the hypothesis that combining those drugs could bring associated benefits. Studies have associated oxidative stress parameters and FMS symptoms, showing a strong relationship between redox status and FIQ scores [21]. Thus, we analyzed the effect of repetitive intravenous lidocaine administration on the redox status of FM patients. Lidocaine/pregabalin could improve the total antioxidant capacity, mainly by upregulating the glutathione (GSH) levels compared to the GPL and GP groups. The tripeptide GSH contains the thiol group that protects the organism from oxidative stress by modulating the enzyme glutathione peroxidase [22]. It could be a biomarker of improved redox balance in FM patients [23].

Those reduced thiol group levels deteriorate in FM patients and the thiol-disulfate rate increases in favor of disulfide amounts. Increased GSH levels seen in GPL patients could contribute to better physical and psychological response after the lidocaine treatment measured by the FIQ scores. Moreover, increased antioxidant redox systems, such as GSH, protect lipids and membranes from oxidative damage [5,24]. FM includes increased plasma lipoperoxides levels in blood $[25,26]$, resulting in altered membrane fluidity and proteinlipid bilayer, altered membrane potentials and eventual integrity leading to the release of cell organelle contents in extracellular fluid. Besides, lipid peroxidation plays a key role in the central nervous system mechanism of depression, anxiety, cognitive dysfunctions, and pain. All of those symptoms are described in FM patients, so lipid oxidation protection is an important FM therapy target [5,24,27].

Together, our data showed in the experiment that lidocaine/ pregabalin therapy stimulate antioxidant defenses in the blood, reducing oxidative stress and consequently lipid bilayer damage and cell membrane disruption. Attenuating oxidative injury could mitigate FM symptoms. These results could propose intravenous lidocaine injection as a safe treatment that may significantly improve FMS patient's quality of life

\section{Acknowledgments}

This study was supported by the Brazilian Agencies National Council for Scientific and Technological Development (CNPq), Coordination for the Improvement of Higher Education Personnel (CAPES), and Foundation to support Research and Technological Innovation of the State of sergipe (FAPITEC-SE).

\section{References}

1. Cohen H (2017) Controversies and challenges in fibromyalgia: a review and a proposal. Royal National Orthopaedic Hospital, Stanmore, Brockley Hill, Stanmore, Middlesex, HA7 4LP, UK. Ther Adv Musculoskelet Dis 9(5): 115-127.

2. Goldenberg DL (1997) Fibromyalgia, chronic fatigue syndrome, and myofascial pain syndrome. National Library of Medicine. Hospital Newton-Wellesley, Massachusetts. Curr Opin Rheumatol 9(2): 135-143.

3. Hudson JI, Goldenberg DL, Pope HGJR, Keck PE JR, schlesinger L (1992) Comorbidity of fibromyalgia with medical and psychiatric disorders. Biological Psychiatry Laboratory, McLean Hospital, Belmont, Massachusetts 02178. Am J Med 92(4): 363-367.

4. Wolfe F, Anderson J, Harkness D, Bennett RM, Caro XJ, et al. (1997) Health status and disease severity in fibromyalgia: results of a six-center longitudinal study. Arthritis Research Center, and University of Kansas School of Medicine, Wichita 67214, USA. Arthritis Rheum 40(9): 15711579.

5. Ghizal F, Das SK, Mahdi AA (2017) Some oxidative and antioxidative parameters and their relationship with clinical symptoms in women with fibromyalgia syndrome. Department of Biochemistry, King George's Medical University, Lucknow, India. Department of Rheumatology, King George's Medical University, Lucknow, India.nt J Rheum Dis 20(1): 3945.

6. Celik F, Gocmez C, Bozkurt M, Kaplan I, Kamasak K, et al. (2013) Neuroprotective effects of carvacrol and pomegranate against methotrexate-induced toxicity in rats. Department of Anesthesiology, Department of Neurosurgery, Department of Physical Medicine and Rehabilitation, Department of Biochemistry, Department of Neurology, Dicle University, Diyarbakir, Turkey. Eur Rev Med Pharmacol Sci 17(22): 2988-2993.

7. Rúas J, Capdevila A (2017) Political communication today: challenges and threats. Communication \& Society. Universitat Rovira i Virgili, Espanha 30 (3).

8. Affaitati G, Costantini R, Fabrizio A, Lapenna D, Tafuri E, et al. (2011) Effects of treatment of peripheral pain generators in fibromyalgia patients. Pathophysiology of Pain Laboratory, Ce.S.I., G. D’Annunzio Foundation, Department of Medicine and Science of Aging, Chieti University, via Carlo de Tocco n. 3, Chieti, Italy. Eur J Pain 15(1): 61-69.

9. Bennett RM, Russell J, Cappelleri JC, Bushmakin AG, Zlatvas G, et al. (2010) Identification of symptom and functional domains that fibromyalgia patients would like to see improved: a cluster analysis. Oregon Health \& Science University, Portland, Oregon, USA. BMC Musculoskelet Disord.

10. Williamson A, Hoggart B (2005) Pain: a review of three commonly used pain rating scales. J Clin Nurs. Research Nurse (Pain), Birmingham Heartland's and Solihull NHS Trust, Birmingham, UK.

11. Benzie IF, Strain JJ (1996) The ferric reducing ability of plasma (FRAP) as a measure of "antioxidant power": the FRAP assay. Department of Health Sciences, Hong Kong Polytechnic University, Kowloon, Hung Hom, Hong Kong. Human Nutrition Research Group, University of Ulster, Coleraine, Northern Ireland, United Kingdom 239(1): 70-76. 
12. Ellman GL (1959) Tissue sulfhydryl groups. Archives of Biochemistry and Biophysics. From the Biochemical Research Laboratory, The Dow Chemical Company, Midland, Michigan USA 82: 70-77.

13. Draper HH, Hadley M (1990) Malondialdehyde determination as index of lipid peroxidation. Meth Enzymol. Methods Enzymol. Academic Press 186: 421-31.

14. Aebi H (1984) Catalase in vitro. Methods Enzymol. Methods in Enzymology. Academic Press 105: 121-126.

15. Misra HP, Fridovich I (1972) The role of superoxide anion in the autoxidation of epinephrine and a simple assay for superoxide dismutase. F.rom the Department of Biochemistry, Duke University Medical Center, Durham, Xorth Carolina 27710 J Biol Chem 247(10): 3170-3175.

16. Masic D, Liang E, Long C, Sterk EJ, Barbas B, et al. (2018) Intravenous Lidocaine for Acute Pain: A Systematic Review. Loyola University Medical Center, Maywood, Illinois. Pharmacotherapy 38(12): 1250-1259.

17. Frölich MA, Mckeown JL, Worrell MJ, Ness TJ (2010) Intravenous lidocaine reduces ischemic pain in healthy volunteers. Department of Anesthesiology, University of Alabama at Birmingham, Birmingham, AL, USA 35(3): 249-254

18. Staud R, Weyl EE, Bartley E, Price DD, Robinson ME (2014) Analgesic and anti-hyperalgesic effects of muscle injections with lidocaine or saline in patients with fibromyalgia syndrome. Department of Medicine, University of Florida, Gainesville, USA. Eur J Pain pp. 803-812.

19. Simone Azevedo de Zanette, Rafael Vercelino,Gabriela Laste, Joanna Ripoll Rozisky, André Schwertner, et al. (2014) Melatonin analgesia is associated with improvement of the descending endogenous painmodulating system in fibromyalgia: a phase II, randomized, doubledummy, controlled trial. Pain and Palliative Care Service at the Hospital de Clínicas de Porto Alegre (HCPA), Universidade Federal do Rio Grande do Sul (UFRGS), Porto Alegre, Brazil. Pharmacology Department, Instituto de Ciências Básicas da Saúde, UFRGS, Porto Alegre, Brazil. Post Graduate Program in Medical Sciences, School of Medicine, Universidade Federal do Rio Grande do Sul (UFRGS), Porto Alegre, Brazil BMC Pharmacol Toxicol. 15: 40.

20. Oliveira MA, Guimarães AG, Araújo AAS, Quintans-Júnior LJ, Quintans JSS (2017) New drugs or alternative therapy to blurring the symptoms of fibromyalgia-a patent review. Multiuser Center for Health (C MultiSaúde), Federal University of Sergipe, Sergipe, Brazil. Departament of Health Education, Federal University of Sergipe, Sergipe, Brazil. Expert Opin Ther Pat 27(10): 1147-1157.

\section{ISSN: 2574-1241}

DOI: 10.26717/BJSTR.2021.39.006261

Lucindo José Quintans Júnior. Biomed J Sci \& Tech Res

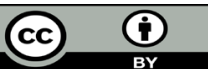

This work is licensed under Creative Commons Attribution 4.0 License

Submission Link: https://biomedres.us/submit-manuscript.php
21. Sarifakioğlu B, Güzelant AY, Güzel EC, Güzel S, Kiziler AR (2014) Effects of 12-week combined exercise therapy on oxidative stress in female fibromyalgia patients. Department of Physical Medicine and Rehabilitation, School of Medicine, Namik Kemal University, 100, Yll Mah, Barbaros Cad. Merkez, Tekirdağ, Turkey. Rheumatol Int 34: 13611367.

22. Dickinson DA, Forman HJ (2002) Cellular glutathione and thiols metabolism. Biochem Pharmacol. Department of Environmental Health Sciences, Center for Free Radical Biology, School of Public Health, University of Alabama at Birmingham, 1530 3rd Avenue S, RPHB-317 Birmingham, AL 35294-0022, USA 64(5-6): 1019-1026.

23. La Rubia M, Rus A, Molina F, Del Moral ML (2013) Is fibromyalgia-related oxidative stress implicated in the decline of physical and mental health status? Clin Exp Rheumatol. Department of Experimental Biology, University of Jaén, Campus Las Lagunillas, Jaen, Spain 31(6 Suppl 79): S121-7.

24. Iqbal R, Mughal MS, Arshad N, Arshad M (2011) Pathophysiology and antioxidant status of patients with fibromyalgia. Rheumatol Int. Zoology Department, G. C. University, Lahore, 54000, Pakistan 31(2): 149-152.

25. Akkuş S, Naziroğlu M, Eriş S, Yalman K, Yilmaz N, et al. (2009) Levels of lipid peroxidation, nitric oxide, and antioxidant vitamins in plasma of patients with fibromyalgia. Medical Faculty, Department of Physical Medicine and Rehabilitation, Suleyman Demirel University, Isparta, Turkey. Cell Biochem Funct 27(4): 181-185.

26. Altindag O, Celik H (2006) Total antioxidant capacity and the severity of the pain in patients with fibromyalgia. Department of Physical Medicine and Rehabilitation, Harran University, Sanliurfa, Turkey. Redox Rep 11(3): 131-135.

27. Oliveira MA, Guimarães AG, Araújo AAS, Quintans-Júnior LJ, Quintans JSS (2017) New drugs or alternative therapy to blurring the symptoms of fibromyalgia-a patent review. Multiuser Center for Health (C MultiSaúde), Federal University of Sergipe, Sergipe, Brazil. Departament of Health Education, Federal University of Sergipe, Sergipe, Brazil. Expert Opin Ther Pat 27(10): 1147-1157.

28. Mccleane G (2007) Intravenous lidocaine: an outdated or underutilized treatment for pain? Rampark Pain Centre, Lurgan, Northern Ireland, United Kingdom. J Palliat Med 10(3): 798-805.

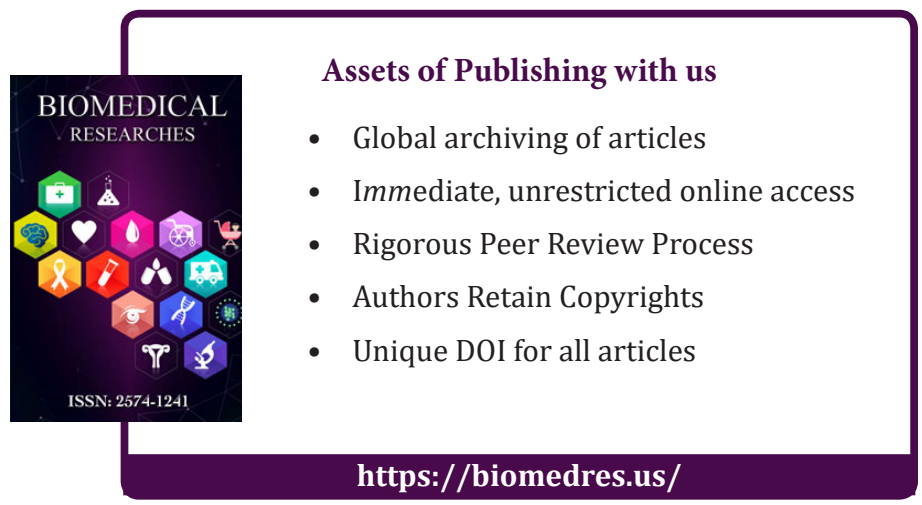

